\title{
REVIEW OF A SPOKE-CAVITY DESIGN OPTION FOR THE RIA DRIVER LINAC*
}

\author{
P.N. Ostroumov ${ }^{\#}$ and K. W. Shepard, ANL, 9700 S. Cass Avenue, Argonne, IL, 60439, USA \\ J.R. Delayen, TJNAF, 12000 Jefferson Avenue, Newport News, VA 23606, USA
}

\section{Abstract}

A design option for the $1.4 \mathrm{GV}$, multiple-charge-state driver linac required for the U. S. Rare Isotope Accelerator Project based on $345 \mathrm{MHz}$, 3-cell spokeloaded cavities has been previously discussed [1]. This paper updates consideration of design options for the RIA driver, including recent results from numericallymodeling the multi-charge-state beam dynamics and also cold test results for prototype superconducting niobium three-spoke-loaded cavities.

\section{INTRODUCTION}

In a previous paper [1], the applicability of TEMclass spoke cavities to high-energy ion linacs was discussed and detailed designs for two TEM-class, triplespoke-loaded niobium resonant cavities covering a velocity range of $0.4 \mathrm{c}<\mathrm{v}<0.75 \mathrm{c}$ were presented.

As a result of their distinct electromagnetic properties and lower frequency, the spoke-loaded cavities were anticipated to offer several advantages over the higherfrequency elliptical-cell cavities in this velocity range.

Since then, two prototype spoke-loaded cavities, operating at $345 \mathrm{MHz}$ and optimized for velocities of $0.5 \mathrm{c}$ and $0.62 \mathrm{c}$, have been under development. Experimental results to date have confirmed the benefits that these cavities could offer. In the particular application to the

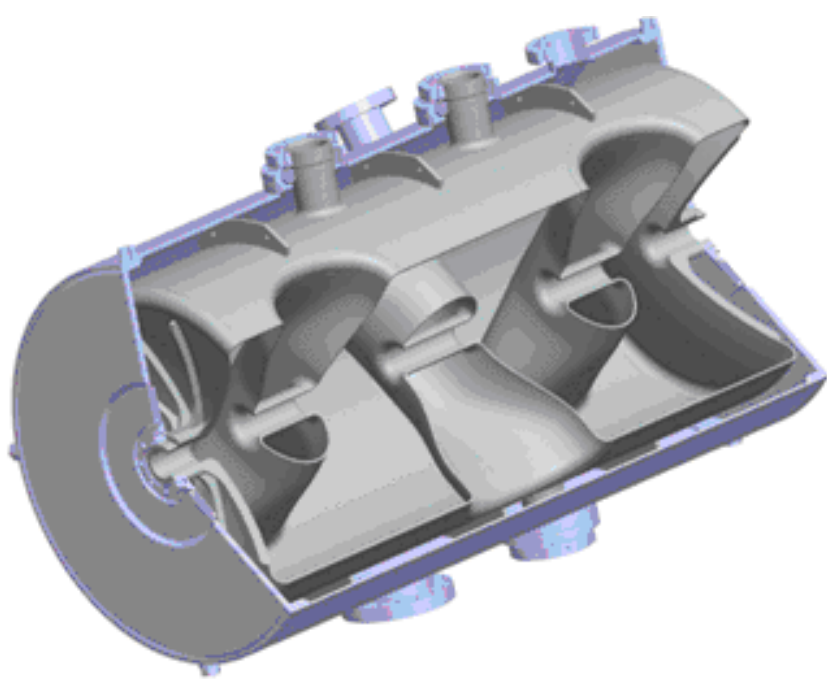

Figure 1: Cut-away view of the design for a niobium triplespoke cavity nested in an integral stainless-steel helium vessel $85 \mathrm{~cm}$ in length.

\footnotetext{
* This work was supported by the U.S. Department of Energy under Contracts No. W-31-109-ENG-38 and DE-AC05-84ER40150.

\# ostroumov@phy.anl.gov
}

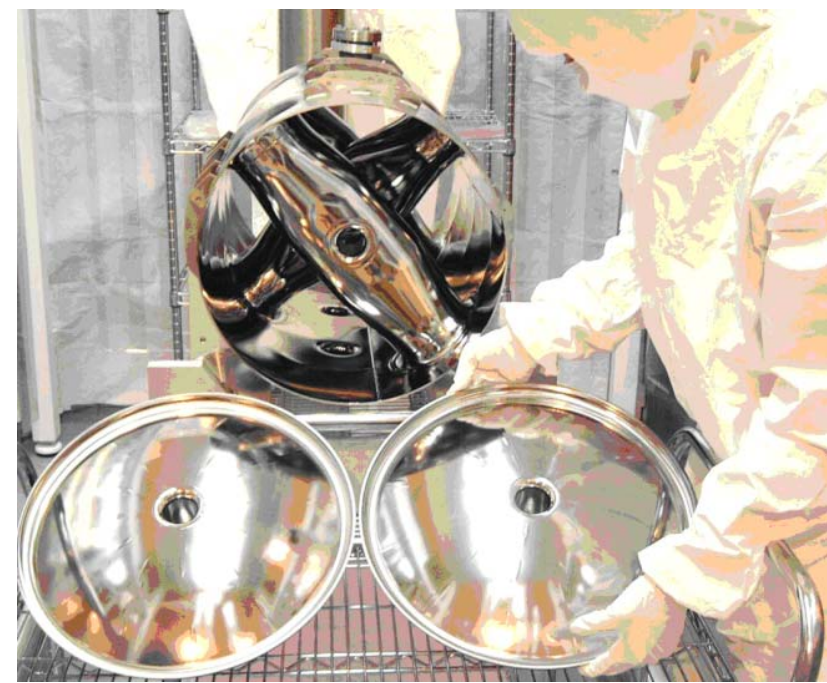

Figure 2: Niobium elements of the prototype superconducting $\beta_{\mathrm{G}}=0.50$ triple-spoke cavity after electropolishing and just prior to welding the complete assembly.

RIA driver, when compared to the measured average performance of the elliptical cavities built for the SNS, they would provide the same voltage gain with similar cryogenic power requirement while eliminating the need for $2 \mathrm{~K}$ operation and reducing the number of cavities. The anticipated very low level of microphonics was also confirmed, thus reducing the amount of rf power required for phase and amplitude stabilization.

Extensive and precise beam dynamic simulations including machine and rf field errors, both static and timedependent, have confirmed the advantages of the larger longitudinal acceptance provided by the lower-frequency spoke cavities. In particular, the tolerances needed to achieve an acceptable level of beam loss and machine activation are significantly relaxed.

In the first part of this paper we present a summary of the experimental results achieved to date on the prototype cavities. In the second part the results of the beam loss analysis for the RIA driver are presented. The third part addresses the applicability of this class of structures to superconducting proton linacs in general, and to the FNAL 8-GeV proton driver in particular.

\section{SUPERCONDUCTING (SC) CAVITIES FOR THE RIA DRIVER LINAC}

Prototypes of two types of SC niobium, $345 \mathrm{MHz}$, three-spoke-loaded cavities with $\beta_{\mathrm{G}}=0.5$ and $\beta_{\mathrm{G}}=0.62$ have been constructed and tested, as detailed in two papers at this conference [2,3]. We summarize here their main features and results, and then compare with 
elliptical-cell cavities developed for the spallation neutron source (SNS) linac [4], and also for RIA [5].

\section{Triple-Spoke Prototype Cavity Test Results}

Figure 1 shows a sectioned view of a design for a three-spoke-loaded, $345 \mathrm{MHz}$ superconducting cavity. Figure 2 shows the major niobium sub-assemblies of the prototype $\beta_{\mathrm{G}}=0.62$ cavity after electropolishing and just prior to completion of the cavity assembly by electronbeam welding.

In recent tests, both the prototype $\beta_{\mathrm{G}}=0.5$ and the prototype $\beta_{\mathrm{G}}=0.62$ triple-spoke cavities were operated $\mathrm{cw}$, at $4.2 \mathrm{~K}$, at peak surface electric fields of more than 27.5 $\mathrm{MV} / \mathrm{m}$, the nominal RIA design goal $[2,3]$. In Table 1 , the results of these tests are compared with recent results for elliptical-cell cavities being developed as another design option for the high-energy section of the RIA driver $[4,5]$.

The $805 \mathrm{MHz}$ elliptical-cell cavities operate at $2 \mathrm{~K}$, while the $345 \mathrm{MHz}$ spoke cavities can be operated at $4.2 \mathrm{~K}$. In order to compare the refrigeration load due to $\mathrm{rf}$ loss into liquid helium, in Table 1 we take 1 watt at $2 \mathrm{~K}$ to be equivalent to 4 watts at $4.2 \mathrm{~K}$ (in terms of total power requirements) [1].

We note that in terms of voltage per cavity and refrigeration load per MV, the spoke cavities are essentially on a par with comparable elliptical cell cavities, even though the development of the spoke cavities is not at this time complete [3].

Because of the relatively small beam-loading of the RIA driver linac, the mechanical stability and microphonic behaviour of the SC cavities are particularly critical. The mechanical properties of the prototype triple spoke cavities are excellent, and microphonic phase-noise under realistic operating conditions is sufficiently small, $\cong$ $1 \mathrm{~Hz} \mathrm{rms}$, to be easily controllable [2].

\section{BEAM LOSS ANALYSIS IN THE RIA DRIVER LINAC}

A detailed design of the RIA driver linac based on triple-spoke resonators (TSR) in the high- $\beta$ section has been developed [1]. In what follows we refer to this option as the triple-spoke linac (TSL). The baseline design of the driver linac is based on an elliptical-cell linac (ECL), the latest update of which was described, for example, in ref. [6]. Both designs of the driver linac have been optimized for simultaneous uranium acceleration with two charge states $\left(28^{+}\right.$and $\left.29^{+}\right)$in the front-end and the first section of the linac up to the first stripper, five charge states between the two strippers (average charge state is $74^{+}$) and five charge states in the high- $\beta$ section (average charge state is $88^{+}$).

Massive parallel-computer end-to-end simulations including machine errors have been performed for both options of the SC driver linac in order to investigate possible beam losses and determine the exact location of these eventual losses in the presence of various machine
Table 1: Comparison of parameters and performance for $805 \mathrm{MHz}$ six-cell elliptical-cell and $345 \mathrm{MHz}$ threespoke-loaded cavities

\begin{tabular}{|c|c|c|c|c|}
\hline \multirow{2}{*}{ Parameter } & \multicolumn{2}{|c|}{ Six-cell Elliptical } & \multicolumn{2}{c|}{ Three-spoke } \\
\cline { 2 - 5 } & $\beta=0.47$ & $\beta=0.62$ & $\beta=0.50$ & $\beta=0.63$ \\
\hline Frequency (MHz) & 805 & 805 & 345 & 345 \\
\hline Length (cm) & 52.7 & 68.2 & 65.2 & 82.15 \\
\hline $\mathbf{E}_{\mathbf{A}}(\mathbf{M V} / \mathbf{m})$ & 10 & 12 & 9.9 & 9.4 \\
\hline $\mathbf{E}_{\mathbf{P E A K}}(\mathbf{M V} / \mathbf{m})$ & 33.4 & 32.5 & 27.5 & 27.5 \\
\hline $\mathbf{B}_{\mathbf{P E A K}}(\mathbf{G})$ & 660 & 572 & 845 & 849 \\
\hline $\mathbf{R} / \mathbf{Q}(\boldsymbol{\Omega})$ & 173 & 279 & 492 & 549 \\
\hline $\mathbf{Q}$ at $\mathbf{E}_{\mathbf{A}}$ & $9.5 \mathrm{E}+09$ & $7.0 \mathrm{E}+09$ & $8.8 \mathrm{E}+08$ & $6.5 \mathrm{E}+08$ \\
\hline Temperature (K) & 2 & 2 & 4.2 & 4.2 \\
\hline $\begin{array}{c}\text { Heat Load* } \\
\text { Watts per MV }\end{array}$ & 12.8 & 16.8 & 14.9 & 21.6 \\
\hline Voltage (MV) & 5.3 & 8.2 & 6.5 & 7.7 \\
\hline
\end{tabular}

* in watts-equivalent at $4.2 \mathrm{~K}$

errors. The details of the simulation technique have been reported recently $[7,8]$.

In a heavy-ion linac one of the major sources of possible particle losses is field errors and misalignments of machine elements. The possible sources of error in the RIA driver linac have been classified into three groups: a) misalignment errors affecting all the elements of the accelerator system: accelerating cavities, quadrupoles, solenoids,... b) rotation errors affecting mainly quadrupoles, multipoles and bending magnets; c) RF field errors affecting the field level as well as the phase of an accelerating cavity.

For heavy ions requiring stripping, another important source of error is the fluctuation in the thickness of the stripper foil or film [7]. The errors are of two types: static and dynamic. Misalignments of accelerator elements are considered as static errors. Jitter of RF and focusing fields are examples of dynamic errors. The phase and amplitude setting of the accelerating cavities when first tuning the accelerator or when restoring a tune is also a source of static errors.

In the presence of misalignments and static rf errors, multiple-charge-state beams in the driver linac require corrective steering and energy correction in order to avoid emittance growth and eventually minimize possible beam losses. To achieve effective steering and energy correction for the whole linac, the latter is divided into 10-15 short sections to which a steering algorithm is applied.

A minimization algorithm has been developed that can correct static errors both in the four-dimensional transverse phase space and longitudinal phase space. The correction of rf static errors is performed by measuring beam energy at the end of the sections and adjustment of accelerating field level in several cavities upstream of the virtual energy measurement device. The algorithm has been fully integrated into the code TRACK [7]. The details of the method related to the correction algorithm in transverse planes can be found in ref. [9]. The beam-based steering and energy correction algorithm is applied to every randomly generated accelerator seed to determine 
the steering correctors setting and adjusted cavity fields along the whole linac for the given set of the element misalignments and static rf errors. The final tracking of large number of particles occurs in the misaligned accelerator with corrective steering applied. Note that without corrective steering a major fraction of the beam would be lost.

The eventual goal of our simulations is to establish tolerances for dynamic and static rf errors for both ECL and TSL options of the driver linac. These tolerances are defined by the level of uncontrolled losses in high-beta section of the linac that must be less than $1 \mathrm{~W} / \mathrm{m}$. In simulations we apply all of the errors simultaneously and study the beam dynamics and the eventual beam losses.

The thickness fluctuation of the stripping foils has been assumed to be $10 \%$ at FWHM. Misalignment errors have the same values as in ref. [7,8] and are kept unchanged in each calculation. Both the baseline and triple-spoke designs of the accelerator were simulated using 200 sets of errors for each combination. For each set, $2 \cdot 10^{5}$ particles are tracked for a total of 40 million particles.

The results of these studies are summarized in Table 2 which show tolerances for static and dynamic RF field amplitude, phase errors for the ECL and TSL options of the RIA driver linac. The uncontrolled beam losses anywhere in the driver linac will be less than $1 \mathrm{~W} / \mathrm{m}$ if errors of rf field and amplitude are less or equal to the numbers given in Table 2.

Table 2: Tolerances for static and dynamic errors of RF field amplitude, phase for the ECL and TSL options of the driver linac.

\begin{tabular}{|l|c|l|}
\hline \multirow{2}{*}{ Option } & $\begin{array}{l}\text { Dynamics RF errors } \\
(\mathbf{r m s})\end{array}$ & Static RF errors \\
\hline \multirow{2}{*}{ ECL } & $0.3 \%, 0.3^{\circ}$ & $\pm 1.5 \%, \pm 1.5^{\circ}$ \\
\cline { 2 - 3 } & $0.5 \%, 0.5^{\circ}$ & $\pm 1.0 \%, \pm 1.0^{\circ}$ \\
\hline \multirow{2}{*}{ TSL } & $0.3 \%, 0.3^{\circ}$ & $\pm 4.0 \%, \pm 4.0^{\circ}$ \\
\cline { 2 - 3 } & $0.5 \%, 0.5^{\circ}$ & $\pm 3.5 \%, \pm 3.5^{\circ}$ \\
\hline
\end{tabular}

\section{APPLICATION OF TRIPLE SPOKE CAVITIES IN SC PROTON LINACS}

Multi-spoke cavities can be effectively applied in the proton/ $\mathrm{H}^{-}$linacs for acceleration from several $\mathrm{MeV}$ to $\sim 400 \mathrm{MeV}$ [10]. In a multi-GeV linac, an acceleration above $400 \mathrm{MeV}$ can be effectively performed by TESLA type cavities operating at $1300 \mathrm{MHz}$. Only one type of triple spoke cavity, operating at the $4^{\text {th }}$ sub-harmonic frequency of $325 \mathrm{MHz}$ with geometrical beta $\beta_{\mathrm{G}}=0.6$, is required for acceleration from $100 \mathrm{MeV}$ to $400 \mathrm{MeV}$.

A frequency jump at $400 \mathrm{MeV}$ which is much higher than in linacs being operated or constructed, significantly improves longitudinal beam dynamics. Figure 3 shows the phase envelope of a $30 \mathrm{~mA}$ beam along an $8-\mathrm{GeV}$ linac where the frequency jump from $325 \mathrm{MHz}$ to $1300 \mathrm{MHz}$ takes place at $400 \mathrm{MeV}$. Selecting a synchronous phase $\sim-30^{\circ}$ immediately after the transition to the $1300 \mathrm{MHz}$ rf

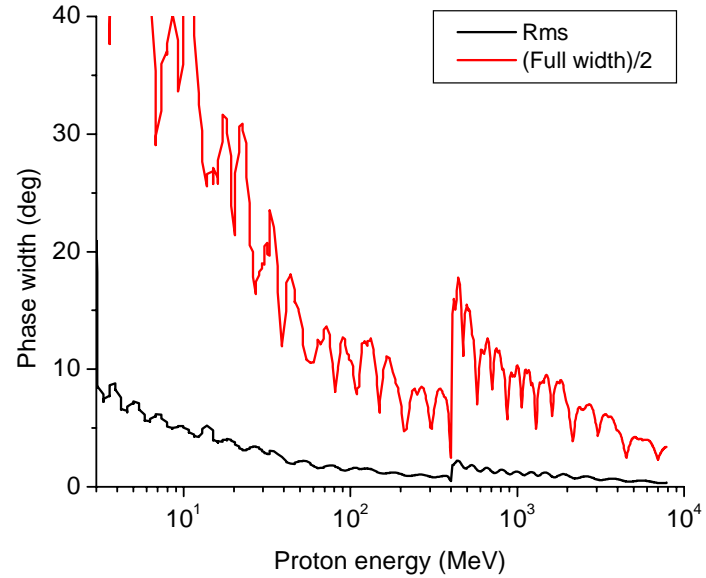

Figure 3: $30 \mathrm{~mA}$ beam phase envelope along the $8-\mathrm{GeV}$ linac simulated for $2 \cdot 10^{5}$ particles without any errors.

structure section provides a large safety margin in the longitudinal phase space.

\section{CONCLUSION}

At the present state of development, spoke-loaded cavities provide similar voltage per cavity and similar refrigeration loads to those obtained with elliptical-cell cavities of more than twice the frequency. The spokeloaded cavities can be operated at $4.2 \mathrm{~K}$ and have excellent mechanical properties.

Massive high-statistics beam dynamics simulations clearly establishes that the triple-spoke option offers much more relaxed error tolerances compared to the baseline design of the RIA driver linac.

\section{REFERENCES}

[1] K.W. Shepard, P.N. Ostroumov, and J.R. Delayen, Phys. Rev. ST Accel. Beams 6, 080101 (2003).

[2] K. W. Shepard, Z. A. Conway, J. D. Fuerst, M. Kedzie, and M. Kelly, Paper TPPT100 in Proc. 2005 Part. Accel. Conf., Knoxville, TN, May 16-20, 2005.

[3] K. W. Shepard, Z. A. Conway, J. D. Fuerst, M. Kedzie, and M. Kelly, Paper TPPT099 in Proc. 2005 Part. Accel. Conf., Knoxville, TN, May 16-20, 2005.

[4] J. R. Delayen, J. Mammosser, and J. P. Ozelis, Paper TPPT079 in Proc. 2005 Part. Accel. Conf., Knoxville, TN, May 16-20, 2005.

[5] C. C. Compton, et al., Phys. Rev. ST Accel. Beams 8, 042003 (2005).

[6] K. Shepard, RIA R\&D Workshop, Washington, D.C., August 26-28, 2003, paper 2.2.3.

[7] P.N. Ostroumov, V.N. Aseev and B. Mustapha. Phys. Rev. ST. Accel. Beams, Volume 7, 090101 (2004).

[8] P.N. Ostroumov, Proc. of LINAC 2004, Lübeck, Germany, August $16-20,2004$, p. 584 (2004).

[9] E. S. Lessner and P. N. Ostroumov. Proc. of the $9^{\text {th }}$ EPAC 2004, Lucerne, Switzerland, 4-9 July 2004, p. 1476.

[10] P.N. Ostroumov, K. W. Shepard and G.W. Foster, Physics Division Annual Report 2003, ANL-04/22, p. 143. 